\title{
Thermal evolution model of 67P/Churyumov-Gerasimenko, the new Rosetta target
}

\author{
M. C. De Sanctis ${ }^{1}$, M. T. Capria ${ }^{1}$, and A. Coradini ${ }^{2}$ \\ 1 Istituto di Astrofisica Spaziale e Fisica Cosmica, Rome, Italy \\ e-mail: mariacristina.desanctis@rm.iasf.cnr.it \\ 2 Istituto di Fisica dello Spazio Interplanetario, Rome, Italy
}

Received 10 March 2005 / Accepted 28 June 2005

\begin{abstract}
Rosetta, the ESA mission dedicated to the detailed study of a comet nucleus before and after the onset of activity, will arrive at comet 67P/Churyumov-Gerasimenko in 2014. At the moment, little is known about this comet, but the successful design of the ROSETTA mission requires some preliminary knowledge of the comet physical parameters. These quantities cannot be determined only through ground-based observations, so predictive models of the thermal evolution and differentiation of the cometary nucleus are necessary. We have developed a comet nucleus thermal evolution model that helps in the estimation of the above quantities. The results have been computed with a numerical code solving simultaneously the heat conduction and gas diffusion equations through an idealized spherical comet nucleus and we show the results obtained applying this model to a comet nucleus with the characteristics of Churyumov-Gerasimenko. Our models indicate that the peculiar dynamical history has a strong effect on the comet: during the orbital stage at large heliocentric distance the comet was covered by a dust crust that is removed when the comet arrives at the present orbit. The computed gas fluxes are in general agreement with those estimated from the observations.
\end{abstract}

Key words. comets: general - comets: individual: 67P/Churyumov-Gerasimenko

\section{Introduction}

Rosetta's main objective is to rendezvous with Comet $67 \mathrm{P} /$ Churyumov-Gerasimenko. It will study the nucleus of the comet and its environment in great detail for a period of nearly two years with an orbiter and a probe on its surface. Rosetta's launch had been originally scheduled for Jan. 2003, but due to the failure of the Ariane Flight 157 in December 2002, the launch was postponed. Rosetta was launched in March 2004 and will meet its new target Churyumov-Gerasimenko in 2014 (the previous one was comet Wirtanen). Comet nuclei are very difficult to observe and no direct data of the interior are at present available: theoretical models of the chemical differentiation and thermal evolution of a nucleus can be used to link coma observations with real nuclei characteristics and properties. Predictive models of the thermal evolution and differentiation of a cometary nucleus are needed to understand the nature of the comet nuclei in terms of composition, structure and physical properties, to understand the physical phenomena that can occur in comet nuclei and foresee comet activity behavior.

Due to the differences particularly in radius and activity between 46P/Wirtanen and 67P/Churyumov- Gerasimenko careful characterization of the comet is important to the success of the ROSETTA mission.

\subsection{Comet 67P/Churyumov-Gerasimenko}

Comet 67P/Churyumov-Gerasimenko, discovered in 1969, has been observed during 6 apparitions: the latest was in 2002-2003. This comet belongs to the Jupiter family: it has a perihelion distance of $1.29 \mathrm{AU}$ and a period of 6.57 years. Unfortunately, only few data on the gas and dust activity have been recorded, because this comet has been poorly observed. The comet radius has been estimated with different techniques. Radar observations (Kamoun et al. 1998) show an upper limit of $3.7 \mathrm{~km}$ for the nucleus radius, while, using HST observations (Lamy et al. 2003) the radius seems to be about $2 \mathrm{~km}$ in agreement with the radius estimated by ground based observations (Tancredi et al. 2000; Kamoun et al. 1998 and Mueller 1992). The observations by Mueller (1992) give a radius of $3.2 \mathrm{~km}$ using an albedo of 0.03. Even if there are some discrepancies in the values of Churyumov-Gerasimenko's size, the data indicate that $67 \mathrm{P} / \mathrm{Churyumov-Gerasimenko} \mathrm{is} \mathrm{one} \mathrm{of} \mathrm{the} \mathrm{largest} \mathrm{Jupiter}$ family comets. This makes it a significantly larger object than the comet 46P/Wirtanen, the initial target of the ROSETTA mission. The estimated rotation period is $\sim 12.3 \mathrm{~h}$.

A limited data set on the production rates is available in the literature. The Lowell Observatory Cometary Database (LOCD) (AHearn et al. 1995; Cochran et al. 1992) reports on $\mathrm{OH}, \mathrm{CN}, \mathrm{C}_{3}, \mathrm{C}_{2}$ and $\mathrm{NH}$, derived from observations during 
the 1982 apparition. Comet 67P/Churyumov-Gerasimenko was found to be depleted in carbon-bearing molecules by Osip et al. (1992) and A'Hearn et al. (1995). Recent observations (Schulz et al. 2004) indicate $\mathrm{C}_{2} / \mathrm{CN}<0.62$ at $2.9 \mathrm{AU}$ : the comet is slightly depleted in $\mathrm{C} 2$ according to the classification scheme introduced by A'Hearn et al. (1995) (the limit is $\mathrm{C}_{2} / \mathrm{CN}<0.66$ ). However, previous observation, in 1982/83 also revealed a depleted abundance ratio $\left(\mathrm{C}_{2} / \mathrm{CN}<0.49\right)$ at a heliocentric distance of about 1.4 AU (A'Hearn et al. 1995). A'Hearn et al. (1995) investigated the $\mathrm{Rh}$ (heliocentric distance) dependence of the $\mathrm{C}_{2}$ and $\mathrm{CN}$, showing that the $\mathrm{CN}$ production rate decreases faster with increasing $\mathrm{Rh}$ than that of $\mathrm{C}_{2}$.

Schulz et al. (2004) reported the postperihelion activity of comet 67P/Churyumov-Gerasimenko between $2.3 \mathrm{AU}$ and 3.2 AU heliocentric distance: the comet showed a very rapid decrease in Af $\rho$ between 2.5 AU and 2.9 AU. The authors interpreted this drop as due to a major change in the outgassing behavior of the comet nucleus that occurred at this part of the orbit.

The comet lightcurves show outbursts at perihelion with strong similarities in the 1982-83, 1996-97 and 2002-2003 apparitions, suggesting that the comet behavior is similar over several returns or with a slight increase with successive passages. A pre/post-perihelion asymmetry with a peak water production of $1 \times 10^{28} \mathrm{~mol} / \mathrm{s}$ has been reported. With the available data it is difficult to say if minor species production rates are constant or peaked around perihelion.

$67 \mathrm{P} /$ Churyumov-Gerasimenko is considered to be a dusty comet (Kiselev 1998) and a peak value of $A f \rho=450 \mathrm{~cm}$ was recorded. The dust coma morphology shows two jet structures in the coma and the orientation of these stationary structures suggests an inclination of the rotation axis of the nucleus of approximately $40^{\circ}$ with respect to the orbital plane (Weiler et al. 2004). Recentetly, Chesley \& Yeomans (2005) estimated the pole of $67 \mathrm{P} /$ Churyumov-Gerasimenko, using the nongravitational acceleration method. They found a pole position RA $=90^{\circ}$ and $\mathrm{Dec}=75^{\circ}$ with an obliquity of about $43^{\circ}$.

The dynamical history of the comet has been studied by Beliaev et al. (1986) and Carusi et al. (1985). They found that the comet had close encounters with Jupiter in 1840 and 1959 that changed the perihelion distance significantly. The comet remained at a quite large perihelion distance between 1840 and 1959 ( $q=2.75 \mathrm{AU})$. The encounter with Jupiter in 1959 reduced the perihelion distance from $q=2.75 \mathrm{AU}$ to the present value $q=1.29 \mathrm{AU}$.

\section{Comet model}

The thermal evolution of a comet nucleus resembling that of 67P/Churyumov-Gerasimenko has been computed with a numerical code solving simultaneously the heat conduction and gas diffusion equations through an idealised spherical comet nucleus (De Sanctis et al. 1999, 2000; Capria et al. 2000a). The comet nucleus is considered initially homogeneous and composed of ices of water, $\mathrm{CO}_{2}$ and $\mathrm{CO}$. The dust component is included as dust particles embedded in an icy matrix. The dust grains are distributed in different size classes and can have different physical and thermal characteristics (mass, den- sity, size, composition, conductivity). Initially, when we start the evolution, the water ice is considered to be in the amorphous phase. The numerical code computes the heat diffusion in the porous cometary material, leading to water ice phase transition and the sublimation of the volatile ices. When the temperature rises, ices start to sublimate, beginning from the more volatile ones: the initially homogeneous nucleus differentiates giving rise to a layered structure, in which the boundary between different layers is a sublimation front. The model takes into account amorphous-crystalline transition with the release of gases trapped in the amorphous ice. The gases diffuse inside the pore system, either re-condensing in the colder layers or escaping in space. The gas flux is computed according to the kinetic theory.

When the ices begin to sublimate the refractory particles become free and are subject to the drag exerted by the escaping gas, so that they can either be blown off or accumulate on the surface to form a crust. To determine how many particles can be blown off and how many can be accumulated on the surface, the different forces acting on the single grain are compared. A critical radius, representing the largest particle that can leave the comet, is obtained for each distribution. At each time step we compute the number of free dust particles and the value of the critical radius: we consider as ejected the grains with radii smaller than the critical one. It must be taken into account that the dust distribution actually observed in the tail of comets can be different to that in the nucleus. The existence of different dust grain size classes allows for differential deposition and differential dust emission. Surface erosion due to ice sublimation, particles ejection, crust formation and compaction are computed at each step. The model is able to describe the nucleus rotation (day/night effects) and the effects of the nucleus obliquity. For further details see previous articles (Capria et al. 2000a,b; De Sanctis et al. 1999; Coradini et al. 1997a,b).

\subsection{Main equations}

Here we recall only the main equations of the thermal evolution model. The heat diffusion through the porous cometary material is described by the following equation, expressing the conservation of energy:

$$
\rho c \frac{\partial T}{\partial t}=\nabla[K \cdot \nabla T]+Q_{\mathrm{H}_{2} \mathrm{O}}+Q_{\mathrm{CO}_{2}}+Q_{\mathrm{CO}}+Q_{\mathrm{tr}}
$$

where $T$ is the temperature, $t$ the time, $K$ the heat conduction coefficient, $\rho$ the density and $c$ the specific heat of the comet material; $Q_{\mathrm{H}_{2} \mathrm{O}}, Q_{\mathrm{CO}_{2}}$ and $Q_{\mathrm{CO}}$ are the specific energies gained or lost by the solid matrix due to sublimation and recondensation of the ices. The terms $Q_{\mathrm{H}_{2} \mathrm{O}}, Q_{\mathrm{CO}_{2}}$ and $Q_{\mathrm{CO}}$ of Eq. (1) are directly linked to the source terms $Q_{x}^{*}$ of Eq. (5):

$$
Q_{x}=-\mathrm{Po} H_{x} Q_{x}^{*} \quad\left[\mathrm{~J} \mathrm{~m}^{-3} \mathrm{~s}^{-1}\right]
$$

where $H_{x}$ is the latent heat of sublimation of component $x(\mathrm{CO}$, $\mathrm{H}_{2} \mathrm{O}$ and $\mathrm{CO}_{2}$ ) and $\mathrm{Po}$ is the porosity.

The term $Q_{\mathrm{tr}}$ accounts for the energy released during the phase transition from amorphous to crystalline ice (Ghormley 1968) according to the activation law experimentally found by Schmitt et al. (1989). 
The gas flow through the pore system is described by the mass conservation equation:

$\frac{\partial \rho}{\partial t}=-\nabla \Phi+Q^{*}$

where $\rho$ is the gas density, $\Phi$ is the flux and $Q^{*}$ the gas source term due to sublimation and recondensation processes.

We assume that the gas density can be related to its pressure through the ideal gas law, and that the flows of different gases do not influence each other: Eq. (3) can be solved separately for each gas. In a quasi-stationary regime, $\Phi$ can be written as

$\Phi_{x}=-G_{x} \nabla P_{x} \quad\left[\mathrm{~mol} \mathrm{~m}^{-2} \mathrm{~s}^{-1}\right]$

where $P_{x}$ is the partial pressure of gas $x$, and $G_{x}$ its gas diffusion coefficient. We assume that the gas temperature is always the same as the matrix through which the gas is moving (the heat capacity of a gas is usually much less than that of a solid). In such a situation, the gas pressure variations do not affect its temperature, and we finally obtain:

$\frac{1}{R T} \frac{\partial P_{x}}{\partial t}=\nabla\left[G_{x} \cdot \nabla P_{x}\right]+Q_{x}^{*}$,

where $R$ is the gas constant.

The model assumes that $Q_{x}^{*}=0$ in all the layers where no ice of the chemical species $x$ is present. Where the ice of gas $x$ is present the gas pressure is considered equal to its saturation pressure, meaning that the gas is always in equilibrium with the solid phase.

The expression for $Q_{x}^{*}$ is:

$Q_{x}^{*}=\frac{1}{R T} \frac{\partial P_{x}}{\partial t}-\nabla\left[G_{x} \cdot \nabla P_{x}\right] \quad\left[\mathrm{mol} \mathrm{m}^{-3} \mathrm{~s}^{-1}\right]$.

The temperature on the surface is obtained by a balance between the solar input and the energy re-emitted in the infrared, conducted in the interior and used to sublimate surface ices.

$$
\begin{aligned}
\frac{S\left(1-A_{\mathrm{s}}\right) \cos Z}{R_{\mathrm{h}}^{2}}= & \epsilon \sigma T_{\mathrm{s}}^{4}+\left.K\left(T_{\mathrm{s}}\right) \frac{\mathrm{d} T}{\mathrm{~d} r}\right|_{r=R_{n}} \\
& +\sum f_{x}^{\mathrm{s}} \cdot H\left(T_{\mathrm{s}}\right)_{x} \cdot \dot{\varepsilon}\left(T_{\mathrm{s}}\right)_{x}
\end{aligned}
$$

where $S$ is the solar constant, $R_{\mathrm{h}}$ the heliocentric distance of the comet, $A_{\mathrm{S}}$ the Bond bolometric albedo of the surface, $\epsilon$ is the infrared emissivity of surface, $\sigma$ the Stefan-Boltzmann constant. The term $Z$ represents the local zenith angle. The term $\cos Z$ is the insolation term that takes into account the spin axis obliquity, the rotation angle and the latitude on the comet surface (Sekanina 1979). In the last term of right hand side, $f_{x}^{s}$ represents the surface fraction covered by different ices, $H$ is the latent heat of sublimation and $\dot{\varepsilon}$ is the sublimation rate.

At the center of the comet the condition is

$$
\frac{\partial T}{\partial r}=0 \text {. }
$$

The surface boundary condition used to solve the diffusion equation Eq. (5) is obtained assuming free sublimation of ice at comet nucleus surface:

$P_{x}=0$.
The center boundary condition for the diffusion equation is obtained by assuming that the ice is in equilibrium with its vapor, and the partial pressures are equal to the saturation pressures at the temperature of the solid matrix.

$P_{x}=P_{x}^{\text {sat }}(T)$.

To determine how many particles can be blown off and how many can be accumulated on the surface, the different forces acting on the single grain are compared, obtaining for each distribution a critical radius that represents the radius of the largest particle that can leave the comet:

$a^{*}=\frac{3}{4} \frac{\Phi_{\mathrm{H}_{2} \mathrm{O}} \cdot V_{\mathrm{H}_{2} \mathrm{O}}+\Phi_{\mathrm{CO}_{2}} \cdot V_{\mathrm{CO}_{2}}+\Phi_{\mathrm{CO}} \cdot V_{\mathrm{CO}}}{\rho_{\mathrm{dust}}\left[G \frac{M_{n}}{R_{n}^{2}}-R_{n} \omega^{2} \cos ^{2} \theta\right]}[\mathrm{m}]$

where $G$ is the gravitational constant, $M_{n}$ the mass of the comet, $R_{n}$ its radius, $\omega$ its angular rotation velocity, $\rho_{\text {dust }}$ the dust grain density, $\Phi_{\mathrm{H}_{2} \mathrm{O}}, \Phi_{\mathrm{CO}_{2}}$ and $\Phi_{\mathrm{CO}}$ are the gas fluxes, and $V_{\mathrm{H}_{2} \mathrm{O}}, V_{\mathrm{CO}_{2}}$ and $V_{\mathrm{CO}}$ the velocities of the gas fluxes. In the Eq. (11) the numerator represents the lifting force exerted by the outflowing gases, and the denominator represents the gravitational attraction corrected by the centrifugal force. All the particles with radius $a<a^{*}$ are blown off and contribute to the dust flux, while those with $a \geq a^{*}$ accumulate on the surface to form the dust crust.

\section{Model parameters}

The composition and structure of comet nuclei are poorly known, and cannot be easily determined from ground observations. Parameters used in these simulations are derived from the observations when available, or chosen among those that are considered typical for comets (Rickman \& Huebner 1990; Huebner et al. 1999). We developed different models to test different parameters and hypotheses (dust distribution, dynamical history, presence of trapped CO). More specifically, we have tested the influence of dust distributions (exponential and Gaussian), of volatile content, orientation and dynamical history (see Tables $1-3$ ).

To understand the influence of the particular dynamical history of this comet a multistage capture process from the Kuiper belt to the final Jupiter family orbit of 67P/ChuryumovGerasimenko is simulated. We used as input the dynamical evolution calculated by Carusi et al. (1985). In one case (Case B) we used a generic multistage process from the Kuiper belt to the present orbit (Table 3).

In the first set of models, we considered the comet rotation axis perpendicular to the orbital plane and we computed our model following four points on the comet surface: $0^{\circ}, 30^{\circ}, 60^{\circ}$ and $85^{\circ}$ of latitude. In the second set of models we used the pole position estimated by Chesley \& Yeomas (2005), that gives an obliquity of $43^{\circ}$.

Different dust size distributions have been used to see the influence of this parameter on the dust flux of the comet. We used three different dust distributions. The Primordial distribution consists of 5 classes from $1 \mu \mathrm{m}$ to $1 \mathrm{~cm}$ with a Gaussian distribution; the Exponential one follows a power index -4 (6 size classes from $1 \mu \mathrm{m}$ to $1 \mathrm{~cm}$ ) with the first class being 
Table 1. Initial parameters.

\begin{tabular}{ll}
\hline \hline Dust/ice & 1 \\
Radius & $2 \mathrm{~km}$ \\
Porosity & 0.6 \\
Rotation period & $12.3 \mathrm{~h}$ \\
Obliquity & $0^{\circ}, 43^{\circ}$ \\
Initial temperature & $30 \mathrm{~K}$ \\
Dust grain thermal cond & $3 \mathrm{~W} / \mathrm{K} / \mathrm{m}$ \\
Dust grain density & $1000 \mathrm{~kg} / \mathrm{m} 3$ \\
$\mathrm{CO}_{2} / \mathrm{H}_{2} \mathrm{O}$ & 0.01 \\
$\mathrm{CO}^{\circ} \mathrm{H}_{2} \mathrm{O}$ & 0.01 \\
\hline
\end{tabular}

Table 2. Model parameters.

\begin{tabular}{ccccc}
\hline \hline Model & Dust distrib. & Dynamic & Trap. CO & Obl. \\
\hline A & primordial & Carusi & yes & $0^{\circ}$ \\
B & primordial & Generic & yes & $0^{\circ}$ \\
C & exponential & Carusi & yes & $0^{\circ}$ \\
D & big grains & Carusi & yes & $0^{\circ}$ \\
N & primordial & Carusi & no & $0^{\circ}$ \\
J & primordial & Carusi & no & $43^{\circ}$ \\
\hline
\end{tabular}

Table 3. Dynamical parameters.

\begin{tabular}{ccccc}
\hline \hline Orbit & $a(\mathrm{AU})$ & $e$ & $q(\mathrm{AU})$ & models \\
\hline m-stage I & 50 & 0.5 & 25 & A,B,C,D,N,J \\
m-stage II & 25 & 0.4 & 15 & A,B,C,D,N,J \\
m-stage III & 8 & 0.5 & 4 & A,B,C,D,N,J \\
67P/C-G (a) & 3.84 & 0.43 & 2.19 & A,C,D,N,J \\
67P/C-G (b) & 4.30 & 0.36 & 2.75 & A,C,D,N,J \\
67P/C-G (c) & 3.51 & 0.63 & 1.29 & A,B,C,D,N,J \\
\hline
\end{tabular}

most numerous; the Big grains distribution has 6 classes, from $1 \mu \mathrm{m}$ to $10 \mathrm{~cm}$ to see the effects of very large grains.

The effect of the kind of volatiles in the nucleus has been evaluated including or excluding the presence of $\mathrm{CO}$ as trapped gas in the amorphous ice. The models have $\mathrm{CO}$ in both forms: $\mathrm{CO}$ ice and $\mathrm{CO}$ trapped in the amorphous ice, but model $\mathrm{N}$ has only $\mathrm{CO}$ ice.

In the second set of models (model J) we have simulated the effects of the obliquity of the pole in the comet evolution. We used the pole position by Chesley \& Yeomas (2005) that determined an obliquity of the spin axis of about $43^{\circ}$.

\section{Model results}

\subsection{Dynamical history}

First, we want to see the influence of the dynamical history on the comet thermal evolution: we make the comparison between model A (our reference), where we assume the Carusi et al. (1985) calculated dynamical path from the Kuiper belt to the present orbit, and model B, where we used a generic multistage

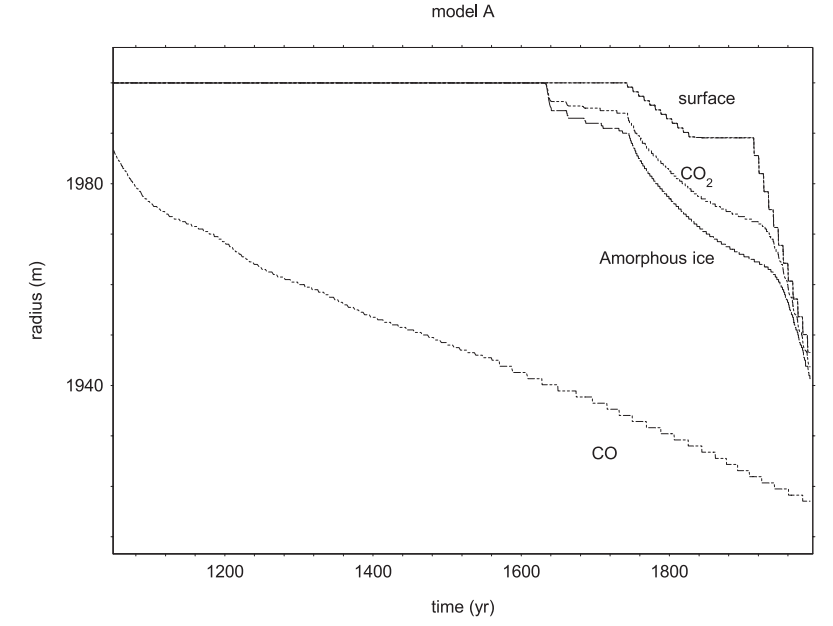

Fig. 1. Model A: nucleus internal evolution during the multistage capture process from the Kuiper belt to the present orbit.

process (the orbits 67P/C-G (a) and (b) of Table 3 are skipped in model B). According to Carusi et al. (1985), this comet has had several close encounters with Jupiter that changed the comet's orbital parameters. In particular, following the calculated dynamical path we see that the comet remained at a large perihelion distance for 100 years $(q=2.75 \mathrm{AU})$ before the close encounter with Jupiter in 1959. The thermal history of this comet strongly affects the overall comet behavior due to the particular orbital evolution. When the nucleus reached the present orbit the internal structure had already been affected by the previous thermal history and the upper layers are already differentiated.

The $\mathrm{CO}, \mathrm{CO}_{2}$ and the amorphous ice are well below the surface due to the progressive heating of the comet that occurred before the last close encounter (Fig. 1). Moreover, the nucleus is covered by a dust crust (Fig. 3). During the orbital phase at large perihelion distance $(q=2.75$ AU before the 1959 close encounter) the comet developed a dust crust. After the 1959 close encounter, with the perihelion distance reduction, the crust was removed and the nucleus re-activated with the ablation of the external layers.

In model B, the nucleus is injected on the final stage after few orbits in a "transition stage" (Multistage III in Table 3). When the comet arrives on the present orbit $(67 \mathrm{P} / \mathrm{C}-\mathrm{G}$ (c) in Table 3), the internal structure is not very differentiated (Fig. 2): the $\mathrm{CO}_{2}$ and the amorphous ice levels are not very deep, while the $\mathrm{CO}$ sublimation front is much further inside the nucleus. When the comet starts to reduce its radius due to the strong sublimation, the $\mathrm{CO}_{2}$ ice front and the amorphous ice front tend to reach the surface. After several orbits, the $\mathrm{CO}$ sublimation front arrives near the surface. The effects of the different depths at which the sublimation fronts are during the comet life are visible in the flux (Figs. 4 and 5). At the beginning of the present orbital stage, the volatile activity is peaked and slightly shifted with respect to the perihelion and water flux and $\mathrm{CO}$ and $\mathrm{CO}_{2}$ show similar trends. After several years the $\mathrm{CO}$ flux changes its perihelion dependance: while the $\mathrm{CO}_{2}$ flux remains similar to the initial orbits (variation correlated to the perihelion distance), the CO flux tends to increase, becoming more constant along the orbit (Figs. 4 and 5). These effects are due to the 


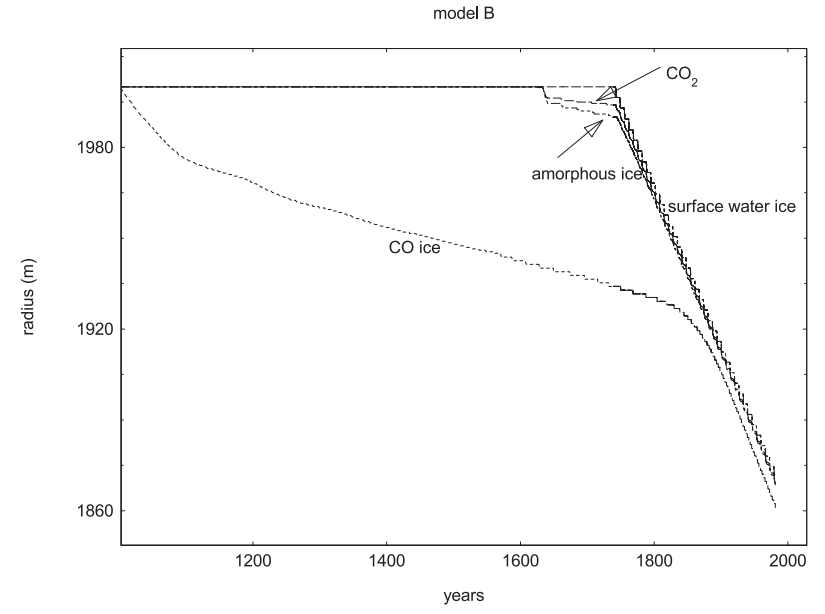

Fig. 2. Model B: nucleus internal evolution during the multistage capture process from the Kuiper belt to the present orbit.

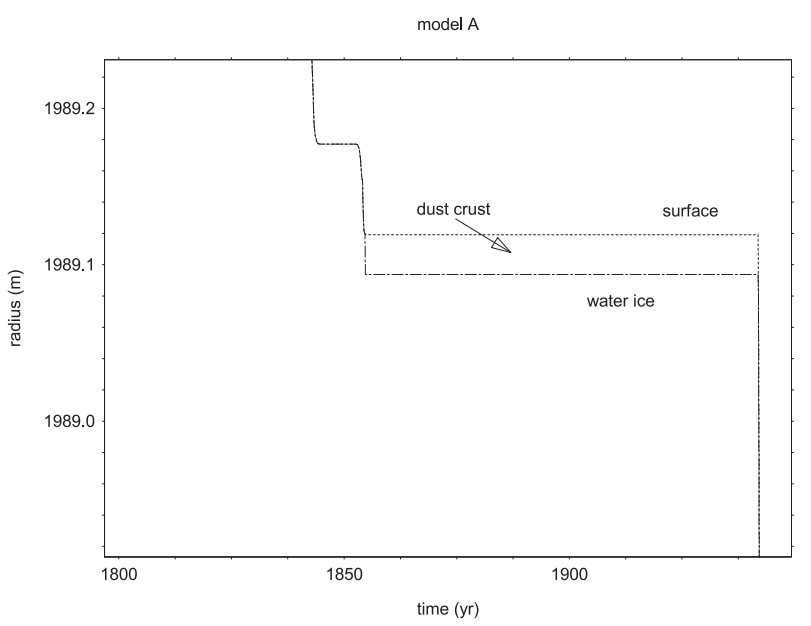

Fig. 3. Model A. Enlarged view of the upper right corner of Fig. 1. The dust crust is on the comet surface.

evolution of the nucleus interior that changes the depth of the different sublimation fronts during its evolution. After a few orbits from the injection in the present dynamical stage, the $\mathrm{CO}_{2}$ sublimation front and the amorphous ice front remain at quite constant depths with respect to the surface. The $\mathrm{CO}$ sublimation front tends to arrive closer to the surface, reaching a quasi-stationary level. The $\mathrm{CO}$ flux at the beginning of this orbital stage is mainly due to the $\mathrm{CO}$ released by the amorphous transition, while, on the last orbits, the $\mathrm{CO}$ flux is the contribution of the $\mathrm{CO}$ sublimation from $\mathrm{CO}$ ice and the $\mathrm{CO}$ coming from the transition. In model $\mathrm{B}$ the nucleus differentiation is less pronounced and, most importantly, there is no crust formation during the evolution. In this case the comet reaches the present orbit without a dust crust on it (Fig. 2).

At the perihelion, for model $\mathrm{A}$ the water flux obtained is $1 \times 10^{21}$ molec s$^{-1} \mathrm{~m}^{-2}$ at the equator (Fig. 6). The gas activity is progressively diminished at high latitude due to the minor solar input (Fig. 7). In polar regions the $\mathrm{CO}$ flux dominates over the others: the $\mathrm{CO}$ is the most volatile species and is able to sublimate also at low temperatures. $\mathrm{CO}$ has a low latent heat and it requires a very low solar energy input to sublimate.

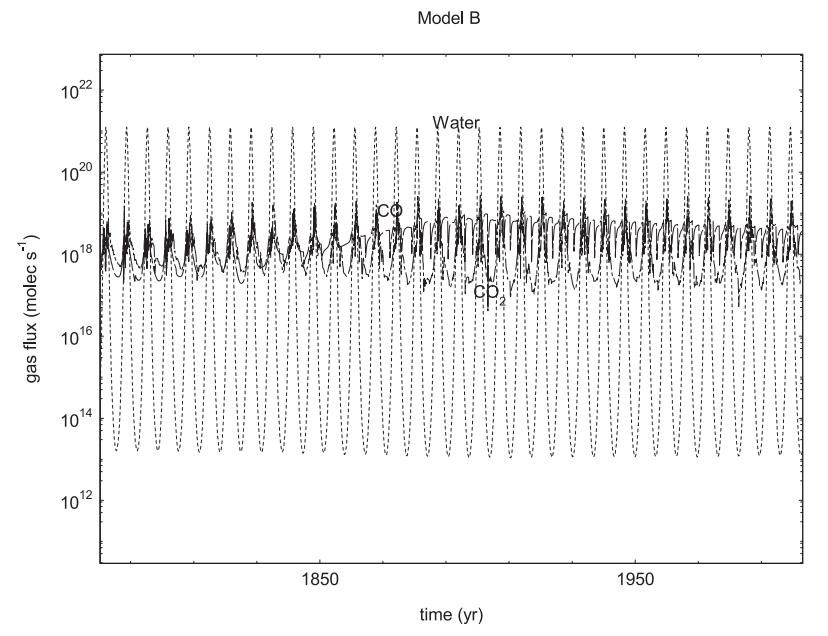

Fig. 4. Model B (present orbit). Water, $\mathrm{CO}$ and $\mathrm{CO}_{2}$ flux versus time.

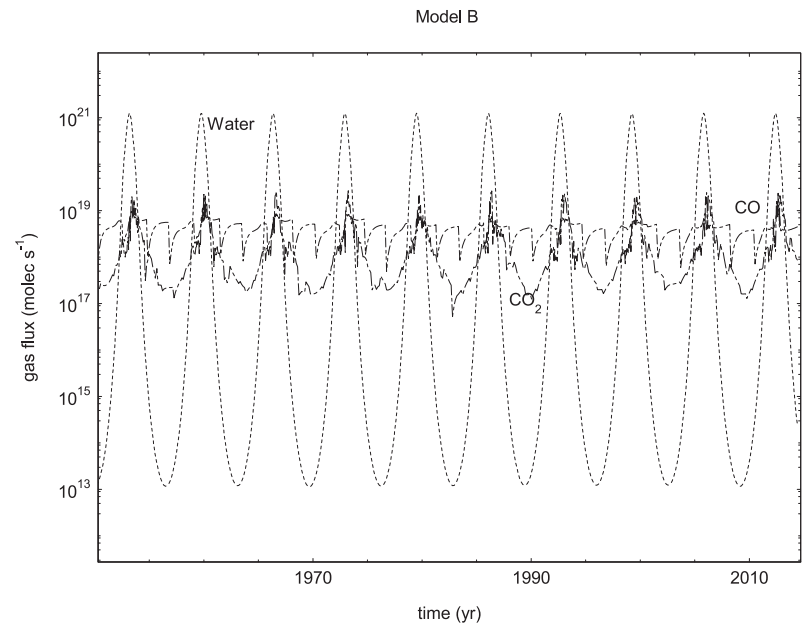

Fig. 5. Model B (present orbit). Enlarged view of model B. Water, $\mathrm{CO}$ and $\mathrm{CO}_{2}$ flux versus time.

If we consider an active surface for a radius of $2 \mathrm{~km}$, scaled for the minor flux at higher latitude, we obtain an overall flux of about $2-3 \times 10^{28} \mathrm{molec} / \mathrm{s}$. Observers report a peak water production of $1 \times 10^{28} \mathrm{molec} / \mathrm{s}$. The water flux shows a large pre/post perihelion asymmetry and steep perihelion distance dependence, similar to that reported by observers. Minor species productions have a less steep perihelion dependence, due to the depth of the volatile sublimation front.

The volatile flux is more pronounced in model B with respect to model A. This is due to the small depth at which the sublimation fronts are located in model B. If we extrapolate model A for a much longer time we can foresee a behavior similar to model B: the surface ablation is such that the ice fronts tend to reach the surface.

When we consider the dynamical path according to Carusi et al. (1985), all the developed models show similar behavior: a long period (1840-1959) of low activity with the formation of a thin dust crust and a slow re-activation after the 1959 change of the orbital parameters. The nucleus is differentiated and this kind of internal structure is common to all sets of models that are developed using the Carusi et al. dynamical evolution. 


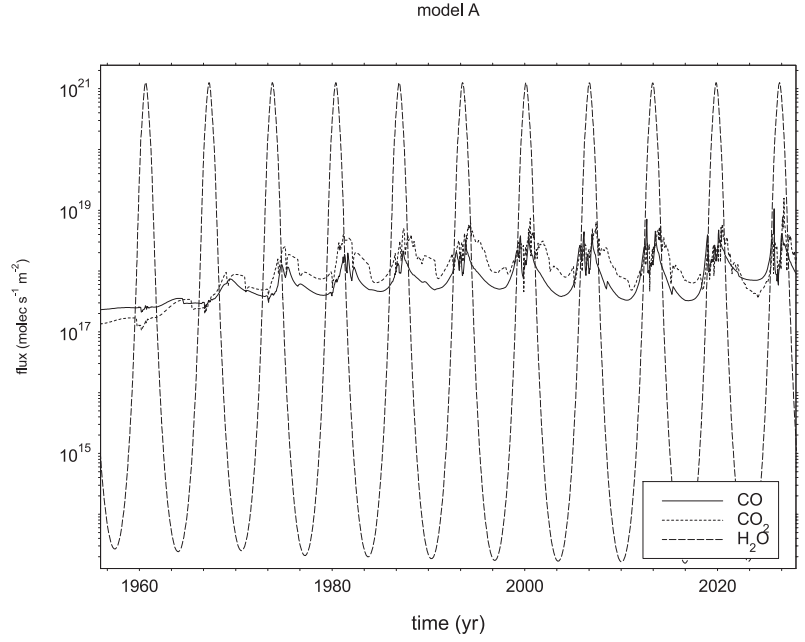

Fig. 6. Model A (present orbit). Water, $\mathrm{CO}$ and $\mathrm{CO}_{2}$ flux versus time.

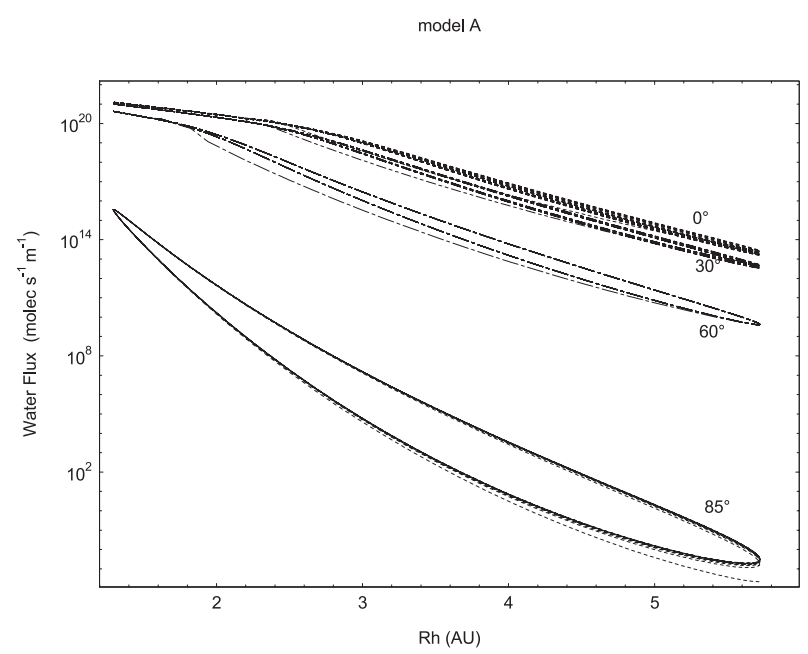

Fig. 7. Model A (present orbit). Water flux versus heliocentric distance computed at different comet latitudes.

\subsection{CO: trapped gas and/or ice}

The influence of the presence of $\mathrm{CO}$ as trapped gas in amorphous ice or as ice has been evaluated with the comparison between model $\mathrm{A}$ and $\mathrm{N}$, where we considered only the presence of $\mathrm{CO}$ in icy form.

In model $\mathrm{N}$ (only $\mathrm{CO}$ ice) the $\mathrm{CO}$ activity is quite constant along the orbit (Fig. 8), while for model A the CO flux follows the perihelion distance. This is due to the fact that, in model $\mathrm{N}$, the $\mathrm{CO}$ ice rich layers are confined below the surface at almost constant temperature. The $\mathrm{CO}$ flux is of the order of $1-2 \times 10^{17}$ molec s${ }^{-1} \mathrm{~m}^{-2}$. The effect of $\mathrm{CO}$ trapped in the amorphous ice is well visible in the flux of model A (Fig. 8). In this case the $\mathrm{CO}$ flux is the contribution of the $\mathrm{CO}$ released during the amorphous-crystalline transition and the $\mathrm{CO}$ sublimating from the $\mathrm{CO}$ sublimation front. The $\mathrm{CO}$ activity follows the perihelion even if the $\mathrm{CO}$ peak is slightly shifted due to the delay of the thermal wave in reaching the deeper layers where amorphous ice is present. In model A the main contributor to the $\mathrm{CO}$ flux is from the release of trapped gas. We can see, also, that as soon as the ablation of the external

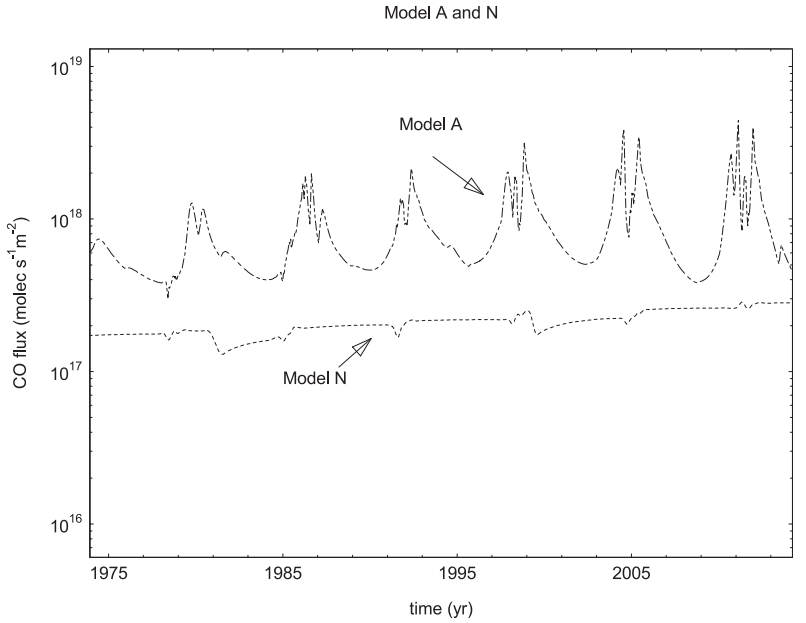

Fig. 8. Model N and model A (present orbit). CO flux versus time.

layers proceeds, the amorphous ice becomes closer to the surface; the time shift of the peak is reduced. The shift is proportional to the distance from the surface of the amorphous ice: the deeper the CO front, the larger the delay in the $\mathrm{CO}$ peak with respect to perihelion. However the differences in $\mathrm{CO}$ flux along the orbit are quite low, the maximum flux at the peak being about $3 \times 10^{18} \mathrm{molec} \mathrm{m}^{-2} \mathrm{~s}^{-1}$ while at the minimum $3 \times 10^{18}$ molec $\mathrm{m}^{-2} \mathrm{~s}^{-1}$.

The only direct information on the $\mathrm{CO}$ emission for this comet are the data acquired in the millimeter wavelengths in May-June 2003, when the comet was at 3 AU from the Sun post perihelion (Bocklee-Morvan et al. 2004). The derived Qco upper limit is about $10^{27} \mathrm{~s}^{-1}$. In both the assumptions (CO trapped as gas in amorphous ice or $\mathrm{CO}$ in icy form) that we made in our simulation, the $\mathrm{CO}$ flux at $3 \mathrm{AU}$ post perihelion is below the upper limit derived by the observation. In the case of model $\mathrm{N}$, our simulation indicates a quite constant flux of about $10^{25} \mathrm{~s}^{-1}$ along the orbit, while in model A the flux at $3 \mathrm{AU}$ is about $10^{26} \mathrm{~s}^{-1}$ assuming a comet radius of $2 \mathrm{~km}$. Based on the available observations, we cannot discriminate between the two kinds of $\mathrm{CO}$ sources.

The nucleus stratification proceeds in a very similar way in model $\mathrm{A}$ and $\mathrm{N}$ and no marked differences emerge in internal structures of the two models. If we analyze the general behavior of the activity for the developed models, we see that the water flux is peaked around the perihelion but it shows large asymmetry pre-post perihelion and the peaks of the minor species, except for model $\mathrm{N}$, are shifted with respect to the perihelion.

\subsection{Dust distribution}

The influence of different dust distributions has been evaluated using three different particles size distributions in models A (primordial distribution), C (exponential distribution) and D (large grain distribution). The overall dust flux behavior is similar in all cases. Dust production shows asymmetry pre/post perihelion. This effect is linked to the water flux asymmetry: the dust flux is driven by the water flux, being much larger than the $\mathrm{CO}_{2}$ and $\mathrm{CO}$ flux. The maximum dust mass loss rate is of the order of $1700 \mathrm{~kg} / \mathrm{s}$. In the case of large grains, model D, 


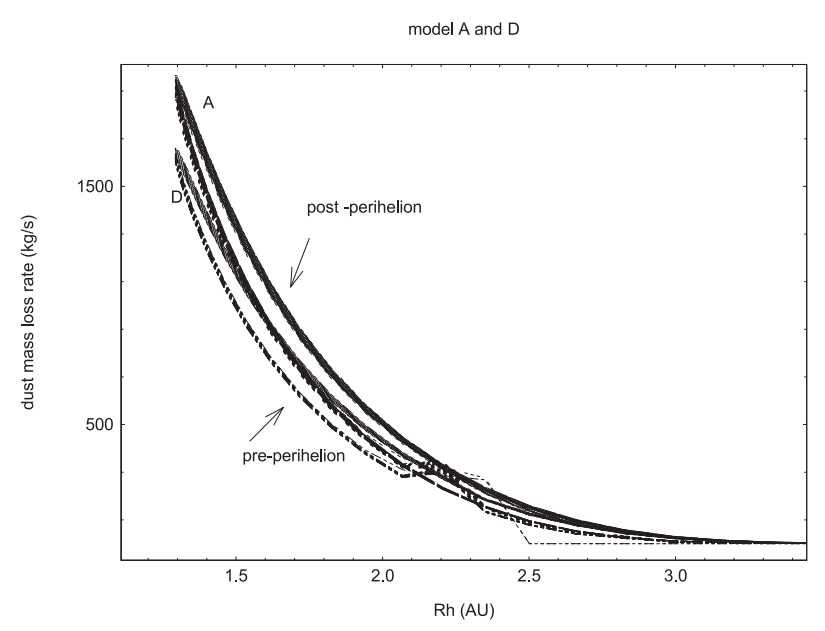

Fig. 9. Dust mass loss rate versus heliocentric distance for models A (primordial dust distribution) and D (large grain dust distribution).

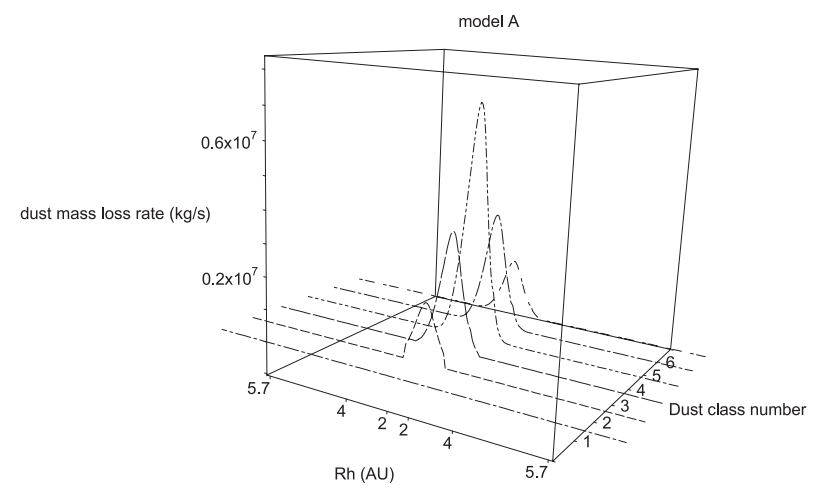

Fig. 10. Model A (present orbit). Number of particles ejected for each size along on orbit: class 6 is the smaller one.

the flux is slightly lower due to the difficulty in blowing off very large grains (Fig. 9). No marked differences are seen when we use the exponential grain size distribution. Most of the particles that are emitted are those of the $10^{-4} \mathrm{~m}$ class (that is the most numerous in model A), but the smaller ones are emitted at larger heliocentric distance (Fig. 10). The dust production rate is in agreement with the estimation by Weiler et al. (2004), who determined a peak of dust of $2000 \mathrm{~kg} / \mathrm{s}$ in 1982 at $1.36 \mathrm{AU}$. However, some observers reported lower dust flux values and different interpretations of the Af $\rho$. Fulle et al. (2004) suggest that the temporal evolution of the dust mass loss rate is opposite to the temporal evolution of the gas loss rate and coma brightness. This could imply seasonal effects and $\mathrm{CO}$ gas flux of at least $5 \times 10^{27} \mathrm{~s}^{-1}$ at very large heliocentric distance (3.6 AU pre perihelion) to sustain the dust particle flux. However, this value of $\mathrm{CO}$ gas flux is not supported by observations that give an upper limit for the $\mathrm{CO}$ of $1.6 \times 10^{27} \mathrm{~s}^{-1}$ at $3 \mathrm{AU}$ after perihelion. Also, the $\mathrm{CO}$ gas flux estimated from our models is about $2 \times 10^{26} \mathrm{~s}^{-1}$, lower then that required by Fulle et al. (2004), and still consistent with the observations.

We want to recall that the dust mass loss rate estimation from observation is derived from the parameter $\operatorname{Af} \rho$, so is model dependent and based on a number of assumptions. For

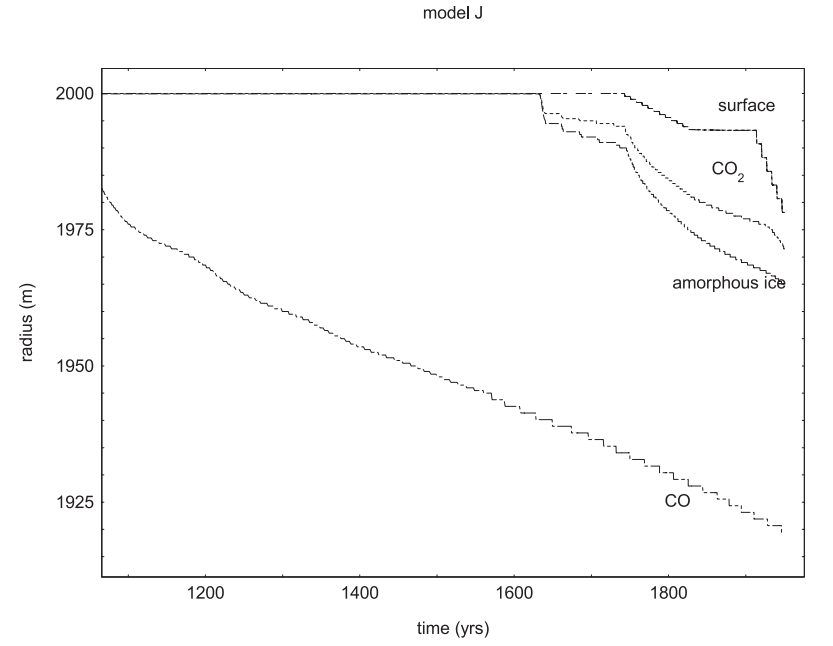

Fig. 11. Model J. Evolution of comet stratigraphy from the Kuiper belt to the present orbit.

this reason the comparison between the computed dust flux and dust flux estimated from observation is not straightforward.

\subsection{Obliquity effects}

In model $\mathbf{J}$ we used the same physical parameters as model $\mathrm{A}$ but adopted the obliquity estimated by Chesley \& Yeomas (2005). We run the model at different latitudes: $0^{\circ}, 30^{\circ}, 45^{\circ}$, $60^{\circ},-30^{\circ},-45^{\circ},-60^{\circ}$. The results obtained indicate that different zones of the comet contribute to the overall flux in different ways with respect to model A, being illuminated to a larger or lesser extent during different parts of the orbit. Also this model develops a dust crust in equatorial zones during the orbital stage at larger perihelion distance. The crust is removed when the comet passes to the present orbit (Fig. 11). In general, for model $\mathrm{J}$, the northern hemisphere at perihelion is the main contributor to the comet activity in term of water flux and dust, while the southern hemisphere contributes to the water and dust flux only during a small period peaked at the perihelion. However, also in this model, the comet does not retain a dust crust easily. In all the models, the activity is "diffuse" even if it is increased in zones of the surface that are preferentially illuminated. If we compare these results with those obtained for the models that do not take into account the estimated obliquity of the rotation axis, we see that the two models (A and $\mathrm{J}$ ) have similar behavior. The maximum water flux is about $2 \times 10^{28}$ around the perihelion. The main difference is in the heliocentric dependance of the water flux: in model $J$ the water follows a less steep Rh dependence until 3.5 AU. Afterwards the decrease of water flux is steeper with respect to model A (Fig. 12).

\subsection{Dust crust}

As stated before, the permanence of a stable dust crust is not foreseen in our Chyurumov-Gerasimenko nucleus models. Formation of a dust crust due only to the sublimation of ices depends on the dynamical age, internal structure and orbital 


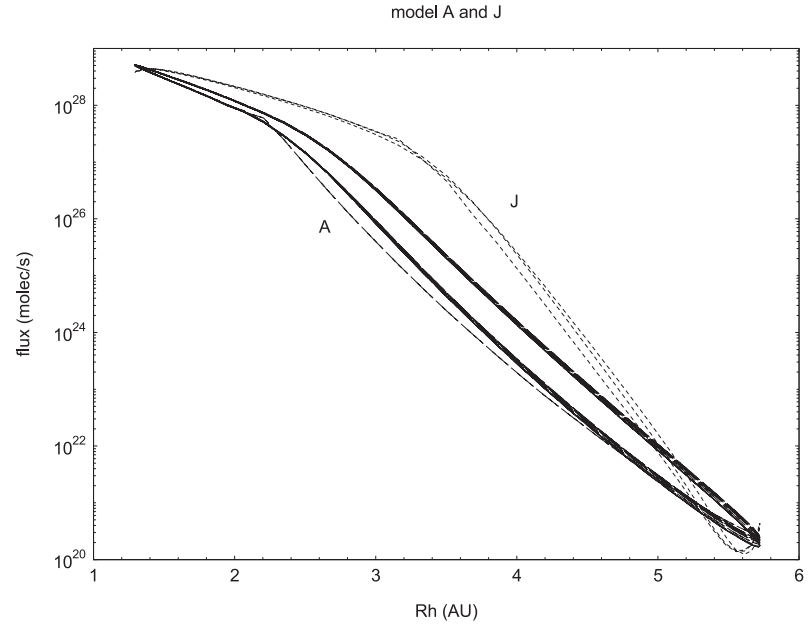

Fig. 12. Model A and J. Water flux versus heliocentric distance.

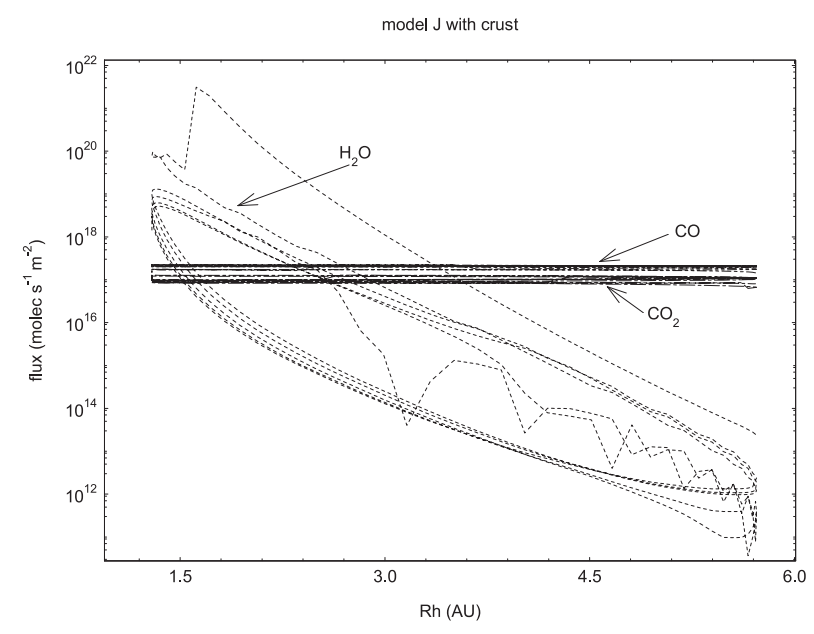

Fig. 13. Water flux from a dust covered regions in model J.

elements of the comet as well as on the physical characteristics of the cometary nucleus. The presence or the absence of a dust crust may be the most important factor that influences the evolution. In all the models we have no permanent crust formation. However we cannot exclude that part of the dust crust can remain on the comet if sticking processes occur. If we want to see the effects of dust crust on the surface we must impose a stable dust crust in our model. In order to favor the formation of a dust crust we have introduced a "trapping" mechanism in the crust formation, as described in De Sanctis et al. (1999). The crust formed with the trapping mechanism is considered porous, so it is possible to have diffuse gas flux from a crust covered region. As an example of the behavior of a non-active zone (covered by a dust crust), we imposed the formation of a crust in model $\mathbf{J}$ at the equator. In Fig. 13 the water flux is shown.

When the crust is formed, the gas molecules cannot flow unobstructed through the dust layer: the mechanism of water ice sublimation changes from the free sublimation regime to the diffusion regime where the gases from the sublimating front diffuse through the crust. The change of regimes can be seen in Fig. 13: as soon as the crust is present the water flux drops by more than a factor of ten. The crust grows slowly, due to the

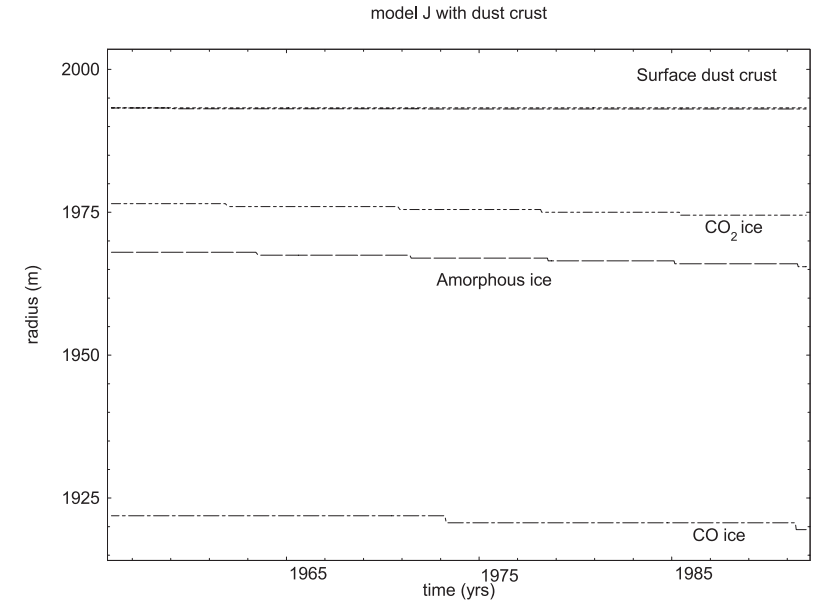

Fig. 14. Model J with dust crust. Internal structure.

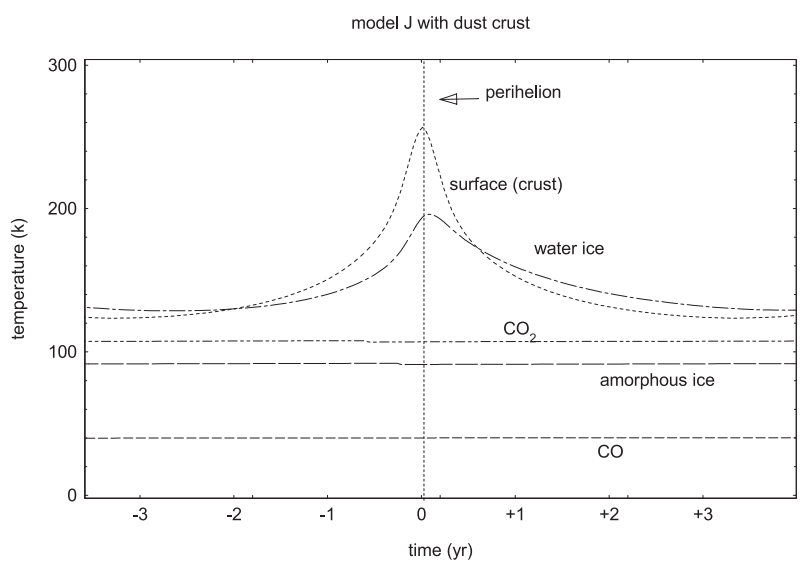

Fig. 15. Model J with dust crust. Surface and ices interfaces temperature (average over a rotation period) during an orbital period.

quenching of activity and the consequent small number of free particles, and tends to reduce the water flux. The water flux in almost 100 times lower with respect to the active surface case.

The internal structure is also affected by the presence of the dust mantle. The levels of the $\mathrm{CO}_{2}$ front and the amorphous ice tend to go down in the nucleus interior, while in the case of no crust these levels tend to stay closer to the surface due to the ablation of the external layers (Fig. 14).

The surface temperature is higher than before, reaching about $260 \mathrm{~K}$ at perihelion on average (over a rotation period) (Fig. 15) with respect to $190 \mathrm{~K}$ (average over a rotation period) in the case of an active surface. The maximum temperature of the water front is shifted with respect to that of the crust because of the time needed for the thermal wave to reach the water ice sublimation front.

\subsection{Night/day differences}

To see how the variation of solar input during comet rotation affects comet evolution, we have modelled the comet rotation assuming a period of $12.3 \mathrm{~h}$. In model $\mathrm{A}$, at the equator and at the perihelion, the maximum surface temperature is $200 \mathrm{~K}$ at noon (day side) while the minimum surface temperature is $145 \mathrm{~K}$ before dawn (night side) (Fig. 16). The layers under the surface 


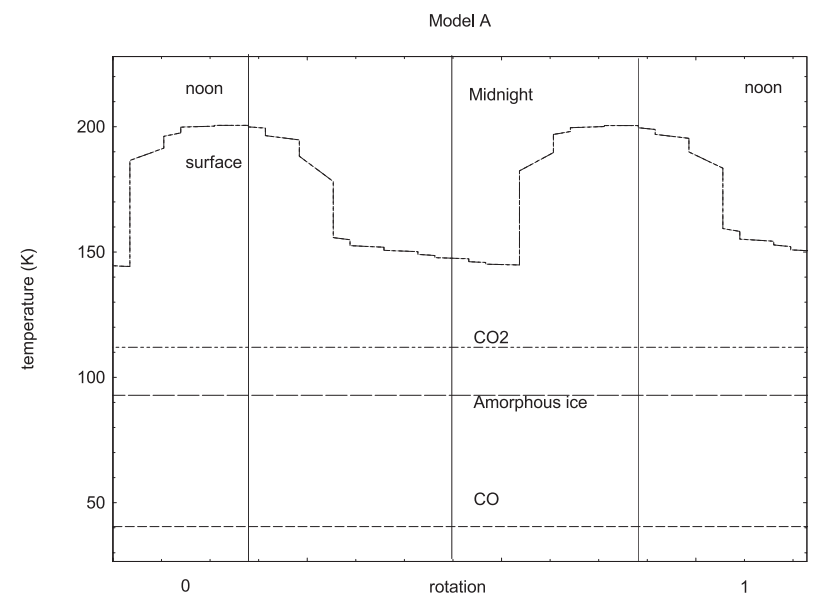

Fig. 16. Model A (present orbit). Night/day surface temperature variation versus rotation.

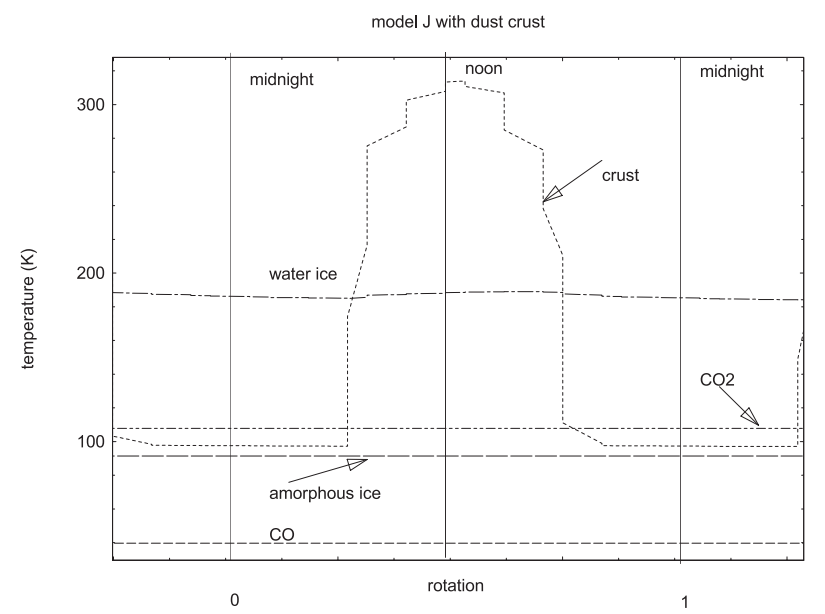

Fig. 17. Model J with dust crust. Night/day surface temperature variation versus comet rotation.

rich in volatiles have constant temperature: the daily thermal wave does not reach those layers. The water and dust production follow the daily temperature variation, while the $\mathrm{CO}$ and $\mathrm{CO}_{2}$ emissions are not affected by the diurnal solar input variation. In this case (model A) the surface is an "active" surface made by a mixture of dust and ice so the surface temperature is referred to this mixed material. Obviously at higher latitude the surface temperature is lower.

We have seen that in the case of a dust crust covering the surface (model $\mathrm{J}$ with a dust crust), the maximum temperature is much higher $(\sim 320 \mathrm{~K})$, while the minimum is about $100 \mathrm{~K}$ (Fig. 17). At the depth where $\mathrm{H}_{2} \mathrm{O}$ sublimates, the daily variation is much less (only a few degrees), and at larger depth, the night/day differences are negligible, indicating that any phenomenon occurring further below the crust is unaffected by the daily change in solar input.

\section{Conclusions}

Our models indicates that the particular dynamical history has a strong effect on the comet. The models foresee the formation of a dust crust during the orbital stage at large perihelion distance, with strong reduction of the overall activity and sinking of the sublimation fronts. When the perihelion distance reduction occurred during the last close encounter (1959) the comet was probably "reactivated". In our models we see a slight increase of the activity passage after passage: the nucleus is progressively ablated and this leads to the reduction of the depth of the sublimation fronts and a slow increase of the activity.

Some observers reported a perihelion asymmetry in the gas activity and Af $\rho$, that they suggested to be caused by seasonal effects (Weiler et al. 2004; Fulle et al. 2004) on an inhomogeneous nucleus surface. However the meaning of this "asymmetry" is not clear. Weiler et al. (2004) say that the increased activity occurred post perihelion but, according to Fulle et al. (2004), the dust flux is larger pre-perihelion even if Af $\rho$ shows the reverse trend, being larger post perihelion. According to Weiler et al. (2004), this comet shows a strong perihelion asymmetry: the enhanced activity postperihelion can be seen in the $\mathrm{CN}$ production rates and Af $\rho$ values. Moreover, the asymmetry in activity seems to be present also for $\mathrm{OH}, \mathrm{C} 2, \mathrm{C} 3$ and $\mathrm{NH}$ in the data, even if the range of heliocentric distances covered by production rates for other species is small (Weiler et al. 2004). Our thermal evolution model is able to explain the perihelion asymmetry in the gas activity without any seasonal effects, but with only the effect of the heat storage in the cometary nucleus. The water flux increase post perihelion is due to the heat stored in the nucleus during the perihelion passage. The volatiles asymmetry is due to the delay of the thermal wave that reaches the deep sublimation fronts: the deeper they are, the larger is the temporal delay and the shift of the activity with respect to the perihelion. The pre/post perihelion asymmetry tends to decrease with the diminishing depth of the sublimation fronts. The observed perihelion dependance and the asymmetry of the minor species, if confirmed by new observations, indicate that the sublimation fronts of the volatiles are close to the surface, as in model A and $\mathrm{J}$, otherwise the $\mathrm{CO}$ flux is quite constant along the orbit, as in model N. However, no direct information on $\mathrm{CO}$ and $\mathrm{CO}_{2}$ are available at the moment.

In the Fulle et al. (2004) scenario the temporal evolution of the mass loss rate is opposite to the evolution of the gas loss rate and the dust coma brightness. According to Fulle et al. (2004), a dust mass loss rate larger at pre-perihelion can be explained as a seasonal effect or also with a homogeneous nucleus where dust fragmentation is active only post perihelion. The main difference between the simulations and the observations is in the interpretation of the dust activity of the comet. In our model the dust loss rate follows the water flux and is peaked around the perihelion.

The general characteristic of all models without an imposed dust crust is the large diffuse activity coming from the subsolar regions that is reflected also in the dust activity. Integrating our perihelion water flux value over the whole nucleus, assumed with a radius of $2000 \mathrm{~m}$ (scaled for the different emission at different latitudes), we obtain a production rate higher than the observed one. Also in the case of a model with obliquity of the rotation axis, the peak of the water activity is similar. This fact could indicate that the comet radius is smaller than $2 \mathrm{~km}$ or that the gas production is limited to some regions of the surface, 
"active regions", while the rest of the comet is covered by dust that quenches the gas flux. However, for all the cases we have simulated, the formation of a stable crust in the present orbit is difficult. The crust is easily formed when the comet is at larger perihelion distance or using a "trapping mechanism". The absence of a stable crust does not mean that on the surface the ice/dust ratio remains constant: this ratio varies continuously and a temporary enrichment of dust with respect to ices is always possible. Moreover, we can speculate that the dust crust formed in the previous stages is not completely removed, resulting in patches of active-non active regions on the comet surface. The high water and dust production rates obtained in our models are easily reduced if we consider that only a small fraction of the comet surface contributes to the gas and dust activity. The observed coma morphology of this comet suggests the presence of areas of enhanced activity on the comet surface. In this scenario the flux expected from the crust-covered regions can be much lower than that from the active regions as suggested by model $\mathrm{J}$ with an imposed dust mantle, but at the moment the exact location and the percentage of active/non active regions is under debate and needs further detailed investigation.

\section{References}

A Hearn, M. F., Millis, R. L., Schleicher, D. G., Osip, D. J., \& Birch, P. V. 1995, Icarus, 118, 223

Beliaev, N. A., Kresak, L., \& Pittich, E. M. 1986, in Catalogue of short-period comets (Bratislava: Slovak Academy of Sciences, Astronomical Institute)

Bocklee-Morvan, D., Moreno, R., Biver, N., et al. 2004, in The new Rosetta targets (Kluver Acd. Pub.), 25

Capria, M. T., Coradini, A., De Sanctis, M. C., \& Orosei, R. 2000a, AJ, 119, 3112

Capria, M. T., Coradini, A., De Sanctis, M. C., \& Orosei, R. 2000b, A\&A, 357, 359
Carusi, A., Kresak, L., Perozzi, E., \& Valsecchi, G. B. 1985, in Long-term evolution of short-period comets Bristol (England and Accord, MA, Adam Hilger, Ltd.), 272

Chesley, S. R., \& Yeomans, D. K. 2005, in Proc. IAU Coll., 197, in press

Cochran, A. L., Barker, E. S., Ramseyer, T. F., \& Storrs, A. D. 1992, Icarus, 98,151

Coradini, A., Capaccioni, F., Capria, M. T., et al. 1997a, Icarus, 129, 337

Coradini, A., Capaccioni, F., Capria, M. T., et al. 1997b, Icarus, 129, 317

De Sanctis, M. C., Capaccioni, F., Capria, M. T., et al. 1999, Planet. Space Sci., 47, 855

De Sanctis, M. C., Capria, M. T., Coradini, A., \& Orosei, R. 2000, AJ, 120,1571

Fulle, M., Barbieri, C., Cremonese, G., et al. 2004, A\&A, 422, 357

Ghormley, J. A. 1968, J. Chem. Phys., 48, 503

Hanner, M. S., Tedesco, E., Tokunaga, A. T., et al. 1985, Icarus, 64, 11

Kamoun, P., Campbell, D., Pettengill, G., \& Shapiro, I. 1998, Planet. Space Sci., 47, 23

Kiselev, N. N., \& Velichko, F. P. 1988, Icarus, 133, 286

Lamy, P. L., Toth, I., Weaver, H., Jorda, L., \& Kaasalainen, M. 2003, AAS/Division for Planetary Sciences Meeting Abstracts, 35

Mueller, B. E. A. 1992, in Asteroids, Comets, Meteors 1991, 425

Osip, D. J., Schleicher, D. G., \& Millis, R. L. 1992, Icarus, 98, 115

Rickman, H., \& Hübner, W. F. 1990, in Physics and chemistry of comets, ed. W. F. Hïner (Berlin: Springer Verlag), 245

Sekanina, Z. 1979, Icarus, 37, 420

Schulz, R., Stüwe, J. A., \& Boehnhardt, H. 2004, A\&A, 422, L19

Schmitt, B., Espinasse, S., Grim, R. A. J., Greenberg, J. M., \& Klinger, J. 1989, in Physics and Mechanics of Cometary Material, ESA-SP 302

Tancredi, G., Fernt'andez, J. A., Rickman, H., \& Licandro, J. 2000, A\&A, 146, 73

Weiler, M., Rauer, H., \& Helbert, J. 2004, A\&A, 414, 749 